\title{
Natural image noise level estimation based on local statistics for blind noise reduction
}

\begin{abstract}
This study proposes an automatic noise estimation method based on local statistics for additive white Gaussian noise. Noise estimation is an important process in digital imaging systems. For example, the performance of an image denoising algorithm can be significantly degraded because of poor noise level estimation. Most of the literature on the subject tends to use the true noise level of a noisy image when suppressing noise artifacts. Moreover, even with the given true noise level, these denoising techniques still cannot attain the best result, particularly for images with complicated details. In this study, a patch-based estimation technique is used to estimate for noise level and applies it to the proposed blind image denoising algorithm. Our approach includes selecting low-rank sub-image with removing high-frequency components from the contaminated image. This selection is according to the gradients of patches with the same statistics. Consequently, we need to estimate the noise level from the selected patches using principal component analysis (PCA). For blind denoising applications, the proposed denoising algorithm integrates the undecimated waveletbased denoising algorithms and PCA to develop the subjective and objective qualities of the observed image, which result from filtering processes. Experiment results depict that the suggested algorithm performs efficiently over a wide range of visual contents and noise conditions, as well as in additive noise. Associated with different conventional noise estimators, the proposed algorithm yields the best performance, higher-quality images, and faster running speed.
\end{abstract}

Keyword: Gaussian noise; Noise estimation; Image denoising; Low-rank patches; PCA 